\title{
Sistema Biomédico para Avaliação e Reabilitação Motora em Hemiparéticos por AVC
}

\author{
Fabrício Noveletto, Pedro Bertemes Filho, Marcelo da Silva Hounsell \\ Centro de Ciências Tecnológicas - Pós-Graduação em Engenharia Elétrica - \\ Universidade do Estado de Santa Catarina (UDESC) - Joinville, SC - Brasil \\ fabricio.noveletto@udesc.br, pedro.bertemes@udesc.br, \\ marcelo.hounsellaudesc.br
}

\begin{abstract}
Hemiparesis resulting from a Stroke can affect the patients' daily activities. In this study, a biomedical system (BS) based on Serious Games $(S G)$ was developed for evaluation and motor rehabilitation in hemiparetic stroke patients, by using a conceptual model for the development of poststroke rehabilitation systems based on the game score. Two experimental studies were performed with twenty-two patients and the results showed that the patients improved significantly in all evaluated clinical variables. The correlations between SG score and clinical assessment scales indicate the potential of BS as a clinical assessment tool.
\end{abstract}

Resumo. A hemiparesia resultante de um Acidente Vascular Cerebral (AVC) pode afetar as atividades de vida diária dos pacientes. Neste estudo foi desenvolvido um sistema biomédico (SB) baseado em Jogos Sérios (JS) para avaliação e reabilitação motora em hemiparéticos por AVC, a partir da proposta de um modelo conceitual para desenvolvimento de sistemas de reabilitação pós-AVC com base na pontuação do jogo. Foram realizados dois estudos experimentais com 22 pacientes e os resultados mostraram melhora significativa em todas as variáveis clínicas analisadas. As correlações encontradas entre a pontuação dos JS e as escalas de avaliação clínica indicam o potencial do SB como uma ferramenta de avaliação clínica.

\section{Caracterização do problema e Motivação}

O Acidente Vascular Cerebral (AVC) é considerado uma das doenças mais incapacitantes. Segundo a Organização Mundial de AVC [Benjamin et al., 2017], a cada ano são registrados cerca de 15 milhões de casos, com um índice de mortalidade de $40 \%$, sendo a segunda principal causa de morte no mundo. Somente no Brasil, no ano de 2011, foram registradas 179 mil internações por AVC, sendo essa uma das doenças crônicas que mais afeta a população brasileira, incidindo principalmente em adultos de meia-idade e idosos [Santos et al., 2011]. A hemiparesia é uma das principais sequelas provocadas pelo AVC, que é caracterizada pela redução da força muscular no lado contralateral à lesão encefálica. Kendall e colaboradores (2007) destacam que a força muscular está diretamente relacionada com a capacidade motora de um indivíduo para realização das atividades da vida diária. As doenças ou síndromes que afetam as funções 
motoras que comprometem a capacidade funcional do indivíduo, como no AVC, geralmente exigem longos períodos de reabilitação [Brewer et al., 2012]. Em muitos casos o processo de reabilitação pode ser permanente, sendo necessário para a manutenção da condição motora do indivíduo. Por isso a importância de buscar estratégias para tornar o processo de reabilitação mais motivador para o paciente. Neste sentido, o uso de jogos digitais tem se mostrado uma importante estratégia na área da reabilitação [Moritz et al., 2011]. Entretanto, embora motivadores, jogos comerciais tradicionais não são desenvolvidos para reabilitação, o que pode limitar a sua eficácia terapêutica. Uma alternativa para esta limitação pode ser o desenvolvimento de jogos para propósitos específicos, também chamados de Jogos Sérios (JS) [Alvarez; Djaouti, 2011]. Vale destacar que a condição de função motora reduzida nestes pacientes também pode limitar o controle do jogo através de interfaces tradicionais e, portanto, uma alternativa mais eficiente, seria combinar o desenvolvimento de JS com interfaces de controle adequadas às necessidades dos pacientes.

A reabilitação é um processo desafiador que exige abordagem multidisciplinar em que novos paradigmas são estabelecidos. A complexidade inerente à reabilitação, principalmente nos processos permanentes, estabelece novas fronteiras que vão além da reabilitação funcional. Por exemplo, os fatores emocionais intrínsecos a algumas doenças são determinantes para o sucesso da reabilitação e exige um esforço contínuo para manter a motivação do paciente. Assim, abordagens que contemplem esses vários aspectos da reabilitação devem ser consideradas. Com base no contexto apresentado, fica evidenciado a importância da reabilitação pós-AVC e a necessidade de novas abordagens que contemplem os aspectos terapêuticos e motivacionais, considerando a característica multidisciplinar da área.

\section{Objetivos e contribuições do trabalho}

A hipótese a ser validada neste trabalho é de que "É possivel utilizar um sistema biomédico baseado em jogo sério para reabilitação motora de pacientes hemiparéticos por AVC por meio de interfaces de controle adaptáveis ao paciente, usando a pontuação do jogo como ferramenta de avaliação clínica." A partir desta hipótese foram elaborados os objetivos geral e específicos.

Objetivo Geral: Desenvolver um sistema biomédico baseado em JS para reabilitação motora de pacientes hemiparéticos por AVC.

Objetivos Específicos: Elaborar um modelo conceitual para desenvolvimento de sistemas biomédicos para reabilitação pós-AVC baseados em JS; Projetar hardware e software com múltiplos sensores para avaliação e reabilitação do equilíbrio e força/controle muscular; Verificar os efeitos terapêuticos do treinamento utilizando JS para equilíbrio e força/controle muscular; Investigar as propriedades métricas dos JS para avaliação de funções motoras com base na pontuação (escore) do jogo;

Neste trabalho é possível destacar algumas importantes contribuições para a área. A primeira contribuição é o modelo conceitual STRONGER (Systems for sTroke RehabilitatioON based on GamE scoRe) para desenvolvimento de sistemas biomédicos para reabilitação do AVC baseados na pontuação (escore) do jogo. O modelo sumariza a relação entre os elementos necessários para o projeto de um JS para reabilitação pósAVC: especialistas, paciente, jogo sério, dispositivo biomédico e escore [Noveletto et 
al., 2018]. A proposta do modelo STRONGER (Figura 1) é pautada no rigor científico e preconiza como elementos fundamentais para a efetividade do JS, os especialistas e o sistema de pontuação. Adicionalmente foi proposta uma taxonomia de JS para reabilitação pós-AVC que pode ser utilizada como um guia dos elementos necessários para o projeto.

Outras contribuições foram o desenvolvimento do sistema biomédico integrado para avaliação e reabilitação motora, que serviu de base para as interfaces de controle adaptáveis de dois JS, um para avaliação e reabilitação do equilíbrio (myBalance) e outro para avaliação e reabilitação de força e controle muscular de membro inferior (mimPong).

A investigação dos efeitos terapêuticos e das propriedades métricas dos JS em pacientes hemiparéticos por AVC é certamente outra importante contribuição deste trabalho.

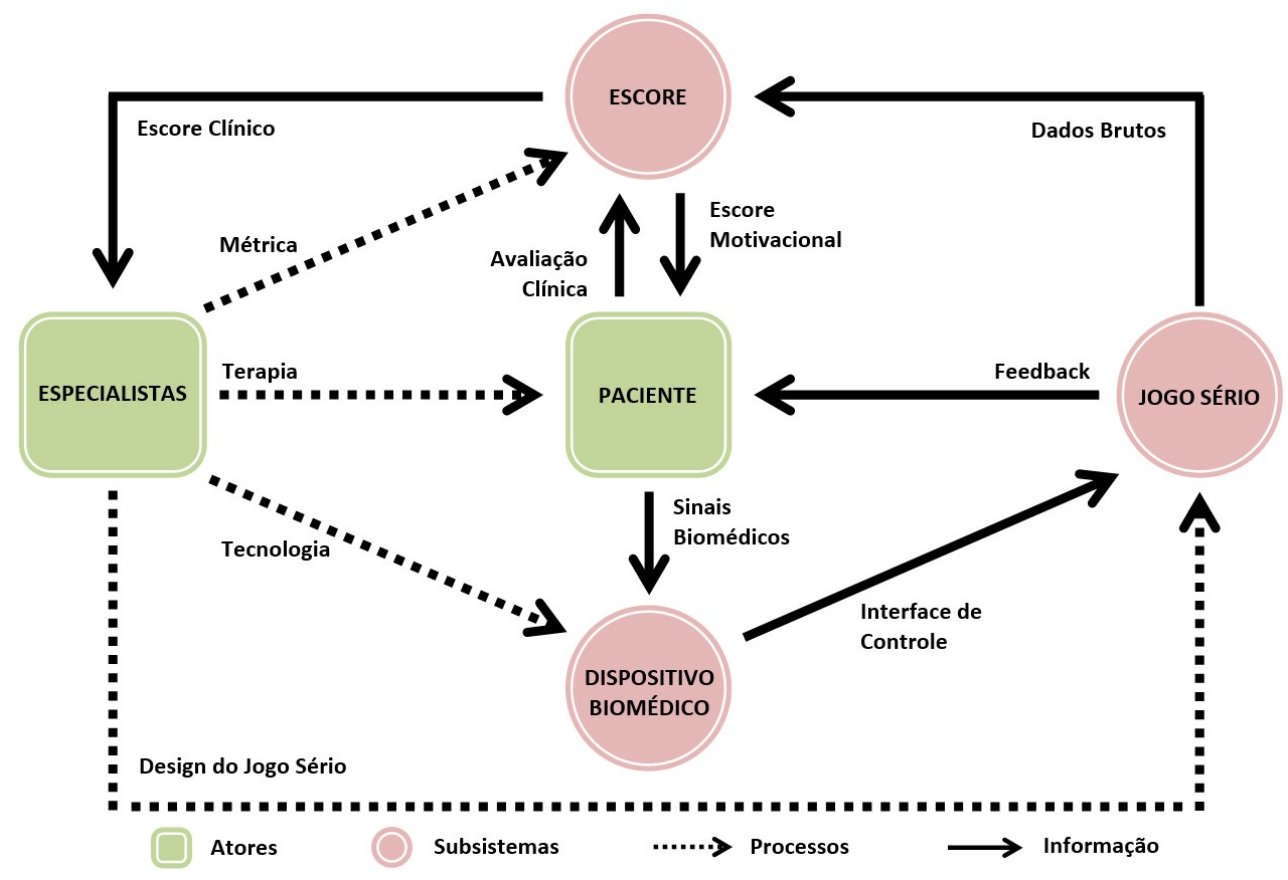

Figura 1. STRONGER: Modelo Conceitual para desenvolvimento de Sistemas de Reabilitação pós-AVC baseados na Pontuação do Jogo.

\section{Resultados e Discussão}

Os principais resultados deste trabalho estão relacionados aos JS myBalance e mimPong, utilizados nas intervenções clínicas com pacientes. Além da eficácia terapêutica, foram avaliadas as propriedades métricas dos JS para utilização como ferramenta de avaliação clínica, correlacionando as pontuações (escores) com as escalas de avaliação motora utilizadas na prática clínica.

\subsection{Sistema Biomédico para Avaliação e Reabilitação do Equilíbrio}

O myBalance é um sistema de JS biomédico para treino e avaliação do equilíbrio que consiste de uma prancha de equilíbrio com sensores inerciais e um sistema computacional que executa o JS. Foi realizado um estudo quase experimental em que 
seis sujeitos hemiparéticos por AVC participaram de um programa de exercícios usando o JS, duas vezes por semana, durante 10 semanas. Doze indivíduos saudáveis, sem disfunção de equilíbrio, foram recrutados para determinar valores referenciais para o equilíbrio usando o sistema proposto. Tamanhos do efeito (Cohen's $d$ ) significativos foram observados em todas variáveis clínicas, com destaque para o equilíbrio dinâmico (EEB), deslocamento do centro de pressão da prancha de equilíbrio (COP) e força do grupo muscular quadríceps femoral do lado afetado (parético). Após o tratamento os pacientes chegaram a um nível de equilíbrio sobre a prancha equivalente aos sujeitos saudáveis. A correlação significativa entre a pontuação do jogo e a escala de equilíbrio de Berg $(r=0,54 ; p=0,001)$ sugere que o uso sistema de pontuação do JS para avaliar o equilíbrio pode ser viável. Os resultados deste estudo reforçam o potencial clínico do sistema proposto para reabilitação do equilíbrio de pacientes hemiparéticos por AVC.

A Tabela 1 mostra os resultados de pré e pós-testes dos pacientes. Foram observadas melhorias estatisticamente significativas em todas as variáveis avaliadas.

Tabela 1 - Valores pré/pós-teste dos pacientes

\begin{tabular}{lrrrr}
\hline Avaliação & Pré & Pós & Valor $\boldsymbol{p}$ & Cohen's $\boldsymbol{d}$ \\
\hline EEB & $45,9(6,6)$ & $51,2(5,2)$ & $<0,001$ & 0,9 \\
TUGT [s] & $21,1(9,6)$ & $17,6(7,6)$ & $<0,001$ & 0,4 \\
PSN & $8,50(9,6)$ & $3,7(4,9)$ & $<0,001$ & 0,6 \\
Força-P [Kgf] & $12,8(4,1)$ & $15,5(5,1)$ & 0,006 & 0,6 \\
Força-NP [Kgf] & $18,9(3,1)$ & $25,0(4,7)$ & $<0,001$ & 1,5 \\
COP [cm] & $121,4(29,7)$ & $76,2(14,0)$ & $<0,001$ & 1,9 \\
Pontuação do JS & $24,3(16.8)$ & $71,9(13,1)$ & $<0,001$ & 3,6 \\
\hline
\end{tabular}

Parâmetros expressos em média e desvio padrão. O tamanho do efeito (Cohen's d) é expresso em módulo. $\mathrm{EEB}=$ Escala de Equilíbrio de Berg; TUGT= Timed Up and Go Test; PSN= Perfil de Saúde de Nothinghan; $\mathrm{COP}=$ Deslocamento do centro de pressão da prancha; Força-P $=$ Força muscular no lado parético; Força-NP = Força muscular no lado não-parético.

\subsection{Sistema Biomédico para Avaliação e Reabilitação Motora de Membro Inferior}

O mimPong é um sistema de JS biomédico para reabilitação do controle e força muscular do membro inferior parético [Noveletto et al., 2018]. O objetivo do JS é controlar uma raquete de tênis, usando a força muscular, para rebater uma bola. Um aparelho extensor de joelho foi adaptado com uma célula de carga e dispositivos de ajuste para medição da força dos grupos musculares extensores (MQF) e flexores do joelho (MIT). Participaram deste estudo quase experimental, 11 sujeitos hemiparéticos por AVC. Um programa de exercícios com o JS foi realizado duas vezes por semana durante três meses (20 sessões de 25 minutos). Um grupo controle com 5 sujeitos hemiparéticos por AVC foi recrutado para um programa de tratamento baseado em cinesioterapia convencional, com o objetivo de comparar os resultados entre as duas abordagens terapêuticas. Os Tamanhos do efeito (Cohen's $d$ ) mostraram que o tratamento com JS foi benéfico para todos os pacientes, sendo superior aos resultados obtidos com a terapia convencional. As correlações significativas entre as pontuações do JS e as escalas de avaliação clínica sugerem que é plausível utilizar a pontuação para avaliar o controle muscular. Especificamente para a escala de recuperação motora (Escala de Fulg-Meyer), as correlações com a pontuação do JS foram de 0,68 (p=0,001) para o grupo MQF e $0,72(\mathrm{p}<0,001)$ para o grupo MIT. 
As Tabelas 2 e 3 mostram os resultados das avaliações de pré e pós-intervenção para o grupo experimental (GE) e grupo controle (GC). Participaram deste estudo um total de 16 indivíduos com hemiparesia crônica decorrente do AVC e que concluíram todas as etapas de avaliação e tratamento, sendo 11 pacientes no GE e 5 pacientes no GC. Os resultados do GC foram utilizados apenas para comparação entre as abordagens terapêuticas (JS e cinesioterapia convencional) e não foram discutidos neste trabalho.

Tabela 2 - Valores de pré e pós-testes das avaliações por escalas clínicas

\begin{tabular}{lrrrr}
\hline Avaliação & Pré & Pós & Valor $\boldsymbol{p}$ & Cohen's $\boldsymbol{d}$ \\
\hline Grupo Experimental (n=11) & & & & \\
EFM-MI & $19,3(4,5)$ & $23,3(3,5)$ & $<0,001$ & 1,0 \\
TUGT [s] & $29,0(16,0)$ & $24,1(14,9)$ & $<0,001$ & 0,3 \\
TVM [m/s] & $0,51(0,34)$ & $0,68(0,51)$ & 0,007 & 0,4 \\
EAM & $1,3(1,2)$ & $0,4(0,8)$ & 0,004 & 0,8 \\
PSN & $14,5(6,6)$ & $10,9(7,6)$ & 0,029 & 0,5 \\
\hline Grupo Controle (n=5) & & & & \\
EFM-MI & $22,6(5,0)$ & $24,0(3,9)$ & 0,135 & 0,3 \\
TUGT [s] & $29,4(21,6)$ & $29,0(21,8)$ & 0,373 & 0,0 \\
TVM [m/s] & $0,65(0,60)$ & $0,66(0,57)$ & 0,822 & 0,0 \\
EAM & $1,8(2,0)$ & $0,4(0,9)$ & 0,135 & 0,9 \\
PSN & $17,4(8,9)$ & $15,8(10,2)$ & 0,477 & 0,2 \\
\hline Pan
\end{tabular}

Parâmetros expressos em média e desvio padrão. EFM-MI= Escala de Fugl-Meyer (membro inferior); TUGT= Timed Up and Go Test; TVM= Teste de Velocidade da Marcha; EAM= Escala de Ashworth Modificada; PSN= Perfil de Saúde de Nothinghan. Cohen's $d$ é expresso em módulo.

Tabela 3 - Valores de pré e pós-testes das avaliações de força muscular

\begin{tabular}{lrrrr}
\hline Avaliação & Pré & Pós & Valor $\boldsymbol{p}$ & Cohen's $\boldsymbol{d}$ \\
\hline Grupo Experimental (n=11) & & & & \\
FMQF-P [kgf] & $13,4(6,5)$ & $17,9(10,9)$ & 0,021 & 0,5 \\
FMIT-P [kgf] & $5,1(2,7)$ & $9,1(4,4)$ & 0,001 & 1,1 \\
FMQF-NP [kgf] & $23,2(5,8)$ & $26,1(11,1)$ & 0,190 & 0,3 \\
FMIT-NP [kgf] & $11,7(4,2)$ & $14,8(5,6)$ & 0,002 & 0,6 \\
\hline Grupo Controle (n=5) & & & \\
FMQF-P [kgf] & $14,7(12,6)$ & $15,8(12,1)$ & 0,070 & 0,1 \\
FMIT-P [kgf] & $7,1(6,6)$ & $8,4(6,8)$ & 0,064 & 0,2 \\
FMQF-NP [kgf] & $24,7(9,8)$ & $26,1(10,3)$ & 0,242 & 0,1 \\
FMIT-NP [kgf] & $13,5(7,7)$ & $14,1(4,4)$ & 0,721 & 0,1 \\
\hline FMQF= Forģ de quán
\end{tabular}

A perspectiva de conseguir reabilitar o paciente, mesmo anos após o AVC, tem motivado a busca de novas estratégias para melhorar e/ou manter as condições funcionais destes pacientes. Nesse sentido, os JS têm se destacado como uma estratégia promissora para a reabilitação pós-AVC. Em geral, o uso de jogos digitais na reabilitação está relacionado com o fator motivacional, que é essencial na reabilitação pós-AVC, pois, em geral, o processo é longo e muitas vezes pode ser permanente, o que favorece levar o paciente a desmotivação e ao abandono da terapia.

Os resultados obtidos em ambos JS indicam que a pontuação do jogo pode representar uma informação com valor clínico. Essa relação é particularmente importante, porque, em geral, os pacientes têm pouca noção de seu progresso na reabilitação [Siegert e Taylor, 2004], e isso é um fator que pode levar ao abandono do 
tratamento. Esta relação ocorre quando existe o equilíbrio entre os aspetos terapêuticos e motivacionais no jogo. Nos projetos do JS mimPong e myBalance, baseados no modelo STRONGER, este balanceamento parece ter sido encontrado, pois os pacientes perceberam esta relação relacionando a sua melhora (clínica) com a melhora na pontuação do jogo.

A evolução clínica dos pacientes atendidos neste estudo é um indicativo de que as abordagens utilizadas foram adequadas e podem ser utilizadas na prática clínica. Atualmente, ambos os JS estão em uso na rotina de atendimento em uma clínica escola de fisioterapia, disponíveis gratuitamente para a comunidade.

\section{Referências}

Alvarez, J; Djaouti, D. (2011) "An introduction to Serious game Definitions and concepts", Serious Games \& Simulation for Risks Management, v. 11, p. 11-15

Benjamin, E. J. et al. (2017) "Heart disease and stroke statistics-2017 update: a report from the American Heart Association", Circulation, 135, n. 10, p. 146-603.

Brewer, L. et al. (2012) "Stroke rehabilitation: recent advances and future therapies", QJM, p. hcs174.

Kendall, F. P.; Mccreary, E.K.; Provance, P.G. (2007) Músculos, provas e funções, Manole, $5^{\mathrm{a}}$ ed.

Moritz, C. et al. (2011) "Neurogame therapy for improvement of movement coordination after brain injury: developing a wireless biosignal game therapy system”. In: Global Humanitarian Technology Conference, IEEE, pp. 72-77.

Noveletto, F. et al. (2016) "Jogo Sério Baseado em Sinal de Força para Reabilitação Motora de Hemiparéticos por AVC”, In XXV Congresso Brasileiro de Engenharia Biomédica.

Noveletto, F. et al. (2018) "Biomedical Serious Game System for Balance Rehabilitation of Hemiparetic Stroke Patients", In IEEE Transactions on Neural Systems and Rehabilitation Engineering, 26(11), 2179-2188.

Noveletto, F. et al. (2018) "STRONGER: A serious game framework for post-stroke rehabilitation", Annals of Physical and Rehabilitation Medicine, v. 61, p. e487.

Santos, D. G. et. al (2011) "Evaluation of functional mobility of patients with stroke sequela after treatment in hydrotherapy pool using the Timed Up and Go Test". Einstein, vol. 9, n. 2, p. 302-6.

Siegert, R. J. e Taylor, W. J. (2004) "Theoretical aspects of goal-setting and motivation in rehabilitation", Disability and rehabilitation, v. 26, n. 1, p. 1-8. 